\title{
A free vascularized fibular graft for reconstruction of mandibular bony defect following excision of odontogenic keratocyst
}

\author{
Farzana B Ibrahim¹, Shamim Hassan², Sajid Hasan ${ }^{3}$, Raihan Anwar' ${ }^{1}$, Md. Rashedul Islam ${ }^{1}$ \\ ${ }^{I}$ Department of Plastic Surgery, BIRDEM General Hospital, Dhaka, Bangladesh; \\ ${ }^{2}$ Department of Plastic Surgery, Khidma General Hospital, Dhaka, Bangladesh; \\ ${ }^{3}$ Department of Maxillofacial Surgery, BIRDEM General Hospital, Dhaka, Bangladesh
}

\begin{abstract}
We present a case of reconstruction of a bony defect due to the excision of recurrent mandibular odontogenic keratocyst in a 45 years old diabetic male. Free vascularized fibular composite graft was taken from the contra lateral lower leg to reconstruct the defect. A two team approach consisting of plastic and maxillofacial (MF) surgeon was adopted. The functional and aesthetical outcome was satisfactory and bone healing occurred without any major complication.
\end{abstract}

IMC J Med Sci 2017; 11(2): 70-72

\section{Introduction}

Cystic and cyst like lesions of the mandible are primarily ellipsoid, radiolucent, and clearly demarcated. It may be odontogenic or nonodontogenic. Odontogenic cysts and tumors develop during or after the formation of teeth [1]. Most odontogenic mandibular lesions are benign, but some particularly odontogenic keratocyst may be locally aggressive and destructive [2]. Odontogenic keratocysts are believed to arise from the dental lamina and other sources of odontogenic epithelium and is highly recurrent and locally aggressive. They represent $5 \%-15 \%$ of all jaw cysts . Most odontogenic keratocysts are found during the 2nd to 4 th decades of life, although they can occur at any age. The lumen of the cyst often contains "cheesy" material and has a parakeratinized lining epithelium [1]. Daughter cysts and nests of cystic epithelia are found outside the primary lesion; as a result, odontogenic keratocysts have the highest recurrence rate $(50 \%)$ of any odontogenic cyst when treated conservatively with curettage [3].

Mandibulectomy has traditionally been the mainstay of surgical therapy for oral squamous cell carcinoma adjacent to or invading the mandible or for osteoradionecrosis. Combined bone grafts are used for large mandible defects $[4,5,6]$. Since microanastomosed bone grafts consist of living tissue, they are capable of independent survival within a compromised recipient site. Furthermore, vascularized grafts are able to improve the local wound regenerative situation. Most commonly used donor sites are iliac crest and fibula. Vascularized fibular grafts present numerous advantages for restoring mandible [3-6]. Their bony architecture is similar to that of the mandible, unlike iliac crest and they are capable of restoring defects up to a length of $25 \mathrm{~cm}$. The grafts can be easily adjusted to the curvature of the mandible using the osteotomy technique. They are associated with very low postoperative donor site morbidity and facilitate the insertion of dental implants [7-9]. Since vascularized grafts behave like an edentulous mandible, osseointegration of dental implants can generally be achieved.

We present a case of a 45 year old diabetic male in whom a free vascularized fibular composite graft from the left leg was used to reconstruct a $6 \mathrm{~cm}$ bony defect of the mandible following excision of odontogenic keratocysts. Informed consent was obtained from the patient to perform this procedure and to publish the case for academic purpose.

Address for Correspondence:

Dr. Farzana B Ibrahim, Registrar, Department of Plastic Surgery, BIRDEM General Hospital, 122, Kazi Nazrul Islam Avenue, Shahbag, Dhaka-1000, Bangladesh. Email: ibrahimfarzana@hotmail.com 


\section{Case report}

A 45 year old, diabetic, male had cyst in the right side of the mandible with a history of repeated infection with pain and discharge for 2 years. Histopathologically the cyst was diagnosed as osteogenic keratocysts. He had history of extraction of lower right first premolar tooth and saucerization for several times but the cyst kept recurring. On examination, his gum was swollen, inflamed and there was absence of lower right $1^{\text {st }}$ premolar tooth [Fig1a]. Radiology showed a large perforation of the cortical plates [Fig 1b, 1c]. Operation was performed under general anesthesia and two team approach was opted. Maxilllo facial surgeons performed the mandibular tumor resection while the plastic surgery team performed the flap and the receptor vessels dissection, and vascular anastomosis. Both teams participated at bone shaping and the final closure.

Maxillofacial team excised about $6 \mathrm{~cm}$ of the mandible keeping the inner and lower cortex intact. The alveolar socket was also excised [Fig 1d]. The radical resection of the tumor was carried out. The plastic surgery team started with harvesting the free fibular composite graft from left leg. The course of the fibula was noted and marked [Fig 1e]. The majority of significant perforators emerge at 10 to $20 \mathrm{~cm}$ below the fibular head, thus it is preferable to locate the skin paddle within this location. As the anterior incision was made through the deep fascia, care was taken to avoid injury to the superficial branch of the peroneal nerve. The dissection continued posteriorly to the posterolateral intermuscular septum, exposing the peroneal muscles. The anterior surface of the septum was then followed down the fibula, and the peroneal muscles were elevated from the lateral and anterior surfaces of the bone. The posterior skin incision was then made through the deep muscle fascia, and the skin paddle was elevated to the edge of the soleus muscle. A $1-\mathrm{cm}$ cuff of soleus muscle was taken from the lateral edge which was later excised. The fibular cuts were made with an oscillating saw. The proximal cut in the fibula was made first and positioned as superiorly as possible without endangering the peroneal nerve. To ensure stability of the knee the proximal $10 \mathrm{~cm}$ of fibula was preserved. Once both cuts were made, the fibula was retracted laterally. The peroneal vessels were located and followed distally where they were ligated and divided.
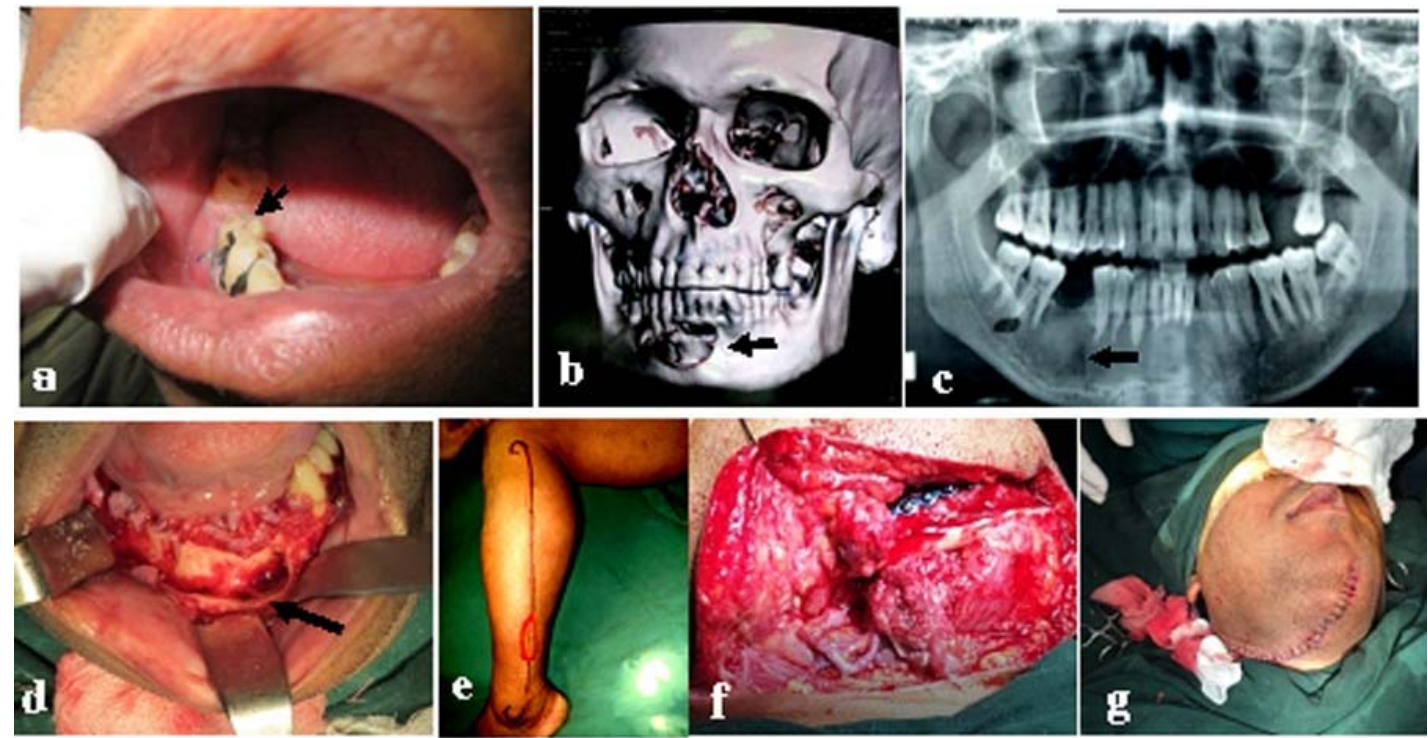

Fig-1: Photograph showing the mandibular bony defect and steps of reconstructive surgery. 1a: Absence of lower right $1^{\text {st }}$ premolar tooth; $1 b$ \& $1 c$ : Large perforation in the cortical plates as shown by $C T$ scan and $X$ ray; $1 d$ : Excised mandible; 1e: The marked course of the fibula for obtaining graft; If \& 1g: Reconstrctuted graft. Arrow indicates the lesions 
The flap dissection continued in a medial to lateral direction to avoid injury to the perforating vessels of the skin. About $8 \mathrm{~cm}$ of fibula was harvested. Once the status of the neck vessels was assured and prepared, the peroneal vessels were divided, and the flap was transferred to the oral defect. A single closing wedge osteotomy was made to create a neoangle and the bone fragments were then stabilized with the rest of the mandible with nail plate and screw fixation. The graft was then revascularized using microvascular techniques [Fig 1f]. We performed peroneal vessel anastomosis to facial artery. Vein anastomosis was performed to the external jugular vein. End-to-end vascular anastomosis was used and microvascular anastomosis was performed as previously described under loupe magnification with 7.0 and 8.0 sutures.

After checking for a watertight intraoral closure, the skin paddle was removed, the neck flaps were replaced, and the skin was closed over drains [Fig 1g]. The leg incision was closed primarily with suction drains in situ. The patient was hospitalized for two weeks. For the first week feeding was allowed with soft diet through naso-gastric tube. Lower limb was immobilized with a cast for the same period. His post operative period was uneventful. Drains and stitches were removed on $5^{\text {th }}$ and $8^{\text {th }}$ post operative day respectively. At three months follow up since the surgical reconstruction, the result was found satisfactory both from oncological and aesthetic point of view.

\section{Discussion}

The free fibular osteofasciocutaneous flap can become the standard option for mandible reconstruction in our experience. The advantages were wide and safe resection and the better functional outcome. The associated morbidity at the donor site was minimal and the technique provided the opportunity of two-team approach thereby reducing the operating time. Immediately after integration dental implantation can be done.

\section{References}

1. Goaz PW, White SC. Oral Radiology: principles and interpretation $3^{\text {rd }}$ ed. St Lois, Mo: Mosby- Year Book, 1994; 398-676.

2. Weber AL. Imaging of cysts and odontogenic tumors of jaw. Radiol Clin North Am. 1993; 31: 101-120.

3. Oikarinen VJ. Keratocyst recurrences at intervals of more than 10 years: case reports. Br J Oral Maxillofac Surg. 1990; 28: 47-49.

4. Hidalgo DA, Pusic AL. Free-flap mandibular reconstruction: a 10-year follow-up study. Plast Reconstr Surg. 2002; 110: 438-451.

5. Hidalgo DA. Fibula free flap: A new method of mandible reconstruction. Plast Reconstr Surg. 1989; 84: 71-79.

6. Sultan MR. Mandible reconstruction with the scapula osteocutaneous flap. Operative techniques in Plastic and Reconstructive Surgery. 1996; 3(4); 248-256.

7. Kuprys R, Varinauskas V, Gervickas A, Stanaityte R. A review of mandibular reconstruction with free microvascularized fibular flap. Sveikatos mokslai/Health Sciences. 2012; 22 3(82): 170-174.

8. Gbara A, Darwich K, Li L, Schmelzle R, Blake F. Long-term results of jaw reconstruction with microsurgical fibula grafts and dental implants. J Oral Maxillofac Surg. 2007; 65(5): 1005-1009.

9. Ferrari S, Bianchi B, Savi A, Poli T, Multinu A, Balestreri A, Ferri A. Fibula free flap with endosseous implants for reconstructing a resected mandible in Bisphosphonate Osteonecrosis. J Oral Maxillofac Surg. 2008; 66(5): 999-1003. 